\title{
Photon-neutrino flux correlations from hadronic models of AGN?
}

\author{
Anita Reimer* \\ Institute for Theoretical Physics, University of Innsbruck, Austria \\ E-mail: anita.reimer@uibk.ac.at
}

\begin{abstract}
Neutrino production in jetted AGN is linked to hadronic processes such as photomeson production. The same interaction predicts also high-energy photons, mostly via neutral pion decay. While neutrinos escape the source unattenuated, the hadronically produced high-energy photons and pairs initiate pair cascades in most cases which re-distribute their energy to lower frequencies where photons can escape the emission region. Realistic hadronic emission models of AGN jets take into account competing energy losses of injected/accelerated particles as well as all leptonic processes (owing to primary and secondary electrons). This may smear out any intrinsic correlation between emerging photon and neutrino fluxes. This work reports on an investigation about the degree of observable photon-neutrino flux and luminosity correlations that is expected from hadronic AGN jet emission models. For this purpose the expected neutrino spectra from a number of hadronically modeled broadband spectral energy distributions (SEDs) of powerful blazars is calculated and compared to the photon fluxes at various frequencies by means of a correlation analysis. The results have implications for the search of the photon sources that are associated to the TeV-PeV neutrino events reported by neutrino observatories.
\end{abstract}

The 34th International Cosmic Ray Conference,

30 July- 6 August, 2015

The Hague, The Netherlands

\footnotetext{
*Speaker.
} 


\section{Introduction}

Energetics arguments consider jetted active galactic nuclei (AGN) as prime candidate sources of the ultra-high energy cosmic rays. If these sources host extremely relativistic nucleons, then hadronic interactions in their dense radiative environments lead inevitably to the production of energetic neutrinos through the decay of the (hadronically) photo-produced charged mesons. It comes therefore to no surprise that blazars (where the almost close line-of-sight alignment of their jets leads to strong beaming enhanced emission) have been proposed as likely cosmic high-energy neutrino sources, and in particular as candidate counterparts of the high-energy neutrino events recently discovered by the IceCube experiment [1].

The kinematics of hadronic interactions in general, and of photomeson production here in particular, predicts a robust relation between the total produced photon-to-neutrino power of roughly 1:1 [7]. Hence, the expected neutrino source and/or diffuse fluxes from $\gamma$-ray bright candidate sources are regularly estimated by employing this relation. However, such procedure is able to provide upper limits at most since competing nucleon energy losses, such as proton synchrotron radiation, can potentially shift large portions of the neutrino power into the photon sector (e.g., [12]). Moreover, the compact blazar emission regions typically proof optically thick for the extremely high photon energies produced in photomeson interactions. Consequently, the hadronically produced photon power is re-distributed over a wide energy regime and can only escape the emission region in large portions when entering the low-opacity energy range. Several bands in the $\gamma$-ray [11], X-ray [6] and radio band [5] have been used in the past for such neutrino flux estimates. This procedure assumes implicitely that the respective energy bands are suitable indicators for the hadronically produced photon flux; proof is still lacking. The present work attempts to improve on this situation by searching for the level of flux and luminosity relations among several currently observationally accessible photon and corresponding neutrino bands in the framework of a current hadronic model [10, 2], with focus on bright $\gamma$-ray blazars (flat-spectrum radio quasars: FSRQs; low- and intermediate-peaked BL Lac objects: LBLs and IBLs).

\section{The model, the sample and its composite model SEDs}

A semi-analytical stationary hadronic blazar model was used in [2] to carry out a comparative leptonic versus hadronic modeling of a sample of 12 bright Fermi-LAT blazars. Here the equilibrium proton distribution has been calculated taking into account the injection of a power-law distribution of proton Lorentz factors $\gamma_{p}$, and losses due to photomeson production, synchrotron radiation and escape. The high-energetic final state particles of hadronic photon-nucleon interactions initiate pair cascades that proceed in the synchrotron-dominated domain. Losses due to synchrotron radiation of charged pions and muons prior to their decay in the magnetized (with field strength $B$ ) emission region have been neglected. This restricts the parameter space that can be explored with this model to those that comply with $B \gamma_{p} \ll$ a few $10^{10} \mathrm{G}$ and $u_{B}>u_{\text {syn }}$ where $u_{\text {syn }}$ is the energy density of the (jet frame) electron synchrotron radiation field and $u_{B}$ is the magnetic field energy density. The selection of the snapshot blazar SEDs (corrected for photon absorption in the extragalactic background light (EBL) using the model of [4]) of the considered sample resulting from this modeling are presented in Fig. 1, along with the associated muon neutrino spectra (cor- 
rected for neutrino oscillations). For this modeling only the jet synchrotron radiation field served as target for particle-photon interactions and cascading. Field strengths in the range $B=10 \ldots 100 \mathrm{G}$ and proton spectra extending up to $\gamma_{p} \sim 10^{8 \ldots 9}$ were used to find suitable model representations of the quasi-simultaneous broadband photon data. The jet remains particle dominated in all cases despite such high field strengths. The SOPHIA Monte Carlo code [8] has then been used for the calculations of the neutrino spectra at source.

Briefly, the sample members were selected according to the following minimum criteria: existence of simultaneous broadband coverage with at least optical/IR, X-ray and $\gamma$-ray data, known source redshift, information on variability and superluminal motion (to derive constraints on modeling parameters), and on accretion disk and BLR luminosity in case of FSRQs. This leads to a sample of particular bright well-known LAT-blazars: 6 FSRQs, 4 LBLs and 2 IBLs are chosen as representatives. As a consequence a prior towards large $>100 \mathrm{MeV}$ photon fluxes but no prior on the neutrino fluxes exists in this sample. The sample covers a redshift $z$ range up to $z=1$ if the most distant sample source, PKS 0528+134 with $z=2.06$, were discarded, and with some source accumulations at $z \sim 0.1,0.3$ and 0.9 .

The broadband SEDs of the modeled blazars typically show two broad humps of non-thermal continuum emission with the low-energy (LE) hump peaking in the optical (FSRQs) to UV frequency range, and explained by electron synchrotron radiation. Hard X-ray photons are either synchrotron photons produced in the HE tail of the primary electrons (preferably for IBLs), or are produced via SSC, proton synchrotron radiation and/or cascade emission (preferably for FSRQs, LBLs). The high-energy (HE) hump shows its characteristic peak energy at $\sim 100 \mathrm{MeV}$ in case of FSRQs, and shifting towards $\sim 1 \mathrm{GeV}$ in the case of BL Lacs. In all sample sources proton synchrotron radiation dominates at their HE peak thereby playing an important role for the production of $\gamma$-rays. Unlike in leptonic models a cascade feature hardens the $\gamma$-ray spectrum beyond its peak, and, in the case of the present sample, dominates the photon emission from FSRQs and BL Lacs at $>100 \mathrm{GeV}$ and $>1 \mathrm{TeV}$, respectively. Owing to large magnetic field strengths the contribution from Synchrotron-Self Compton (SSC) emission is typically small in hadronic AGN models. Still, in selected cases and in a small X-ray range SSC may contribute in shaping the SED.

Pair cascading is a characteristic feature of hadronic AGN emission models, usually negligibly small or non-existent in corresponding leptonic models. For the present sample sources the overall contribution from cascade emission to the HE hump in the present hadronic model SEDs appears on average larger for BL Lacs than for FSRQs. Its extend into the HE regime at source is governed by the $\gamma \gamma$-pair production opacity $\tau_{\gamma \gamma}$ in the emission region. Fig. 2 shows a typical example of the corresponding photon escape probability for the present sample sources. Here, efolding (jet-frame) energies $E^{\prime}\left(\tau_{\gamma \gamma}=1\right)$ lie typically at $\mathrm{TeVs}$, leading to photons with $\leq 1 \mathrm{TeV} \cdot D$ (where $D \sim 10-30$ in the present sample is the bulk Doppler factor) to escape the emission region. However, in all the source SEDs considered here photon absorption in the EBL causes an earlier cutoff of this cascade component towards high energies.

Comparing the overall HE photon and neutrino power in the composite SEDs as shown in Fig. 1 the impact of the strong proton synchrotron component in the hadronic model SEDs is apparent: Photon-to-neutrino power ratios (at source ) are roughly found to lie in the 10-100 range for FSRQs, and 1-10 range for BL Lacs here. This also indicates a fairly high degree of scatter to be expected in the correlation analysis. The neutrino SEDs of the present sample show their power 
peak typically in the $0.1-1 \mathrm{EeV}$ energy range.
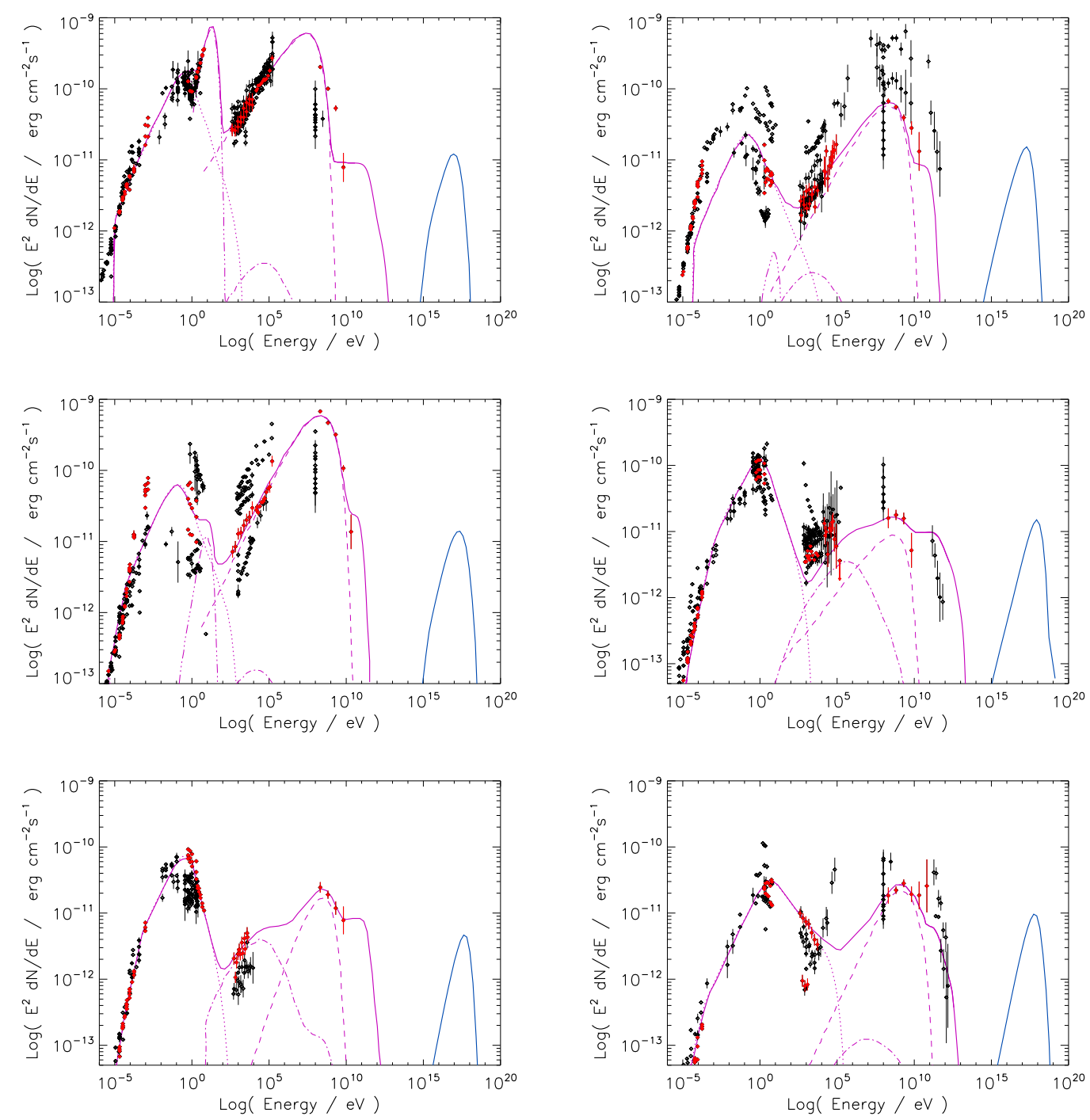

Figure 1: Composite photon (violet curves) and $(\mu+\bar{\mu})$ neutrino (blue solid curve) broadband spectra of 3C 273 (upper left), 3C 279 (upper right), 3C454.3 (middle left), BL Lacertae (middle right), OJ 287 (lower left) and W Comae (lower right). The photon SED consists of electron synchrotron (dotted curve) and proton synchrotron radiation (dashed curve), SSC (dashed-dotted) and accretion disk radiation (dashed-triple-dotted curve). The solid line refers to the sum of those and includes the cascading component. The SEDs has been corrected for absorption in the EBL using the model of [4]. Red data points indicate the quasi-simultaneous data set while black data points are non-simultaneous archival data.

In order to evaluate whether rank order is nevertheless maintained (high photon powers indicating high neutrino powers) a dedicated (rank order) correlation analysis is performed.

\section{Correlation study}

It has frequently been demonstrated in the past that correlating source intrinsic powers such 


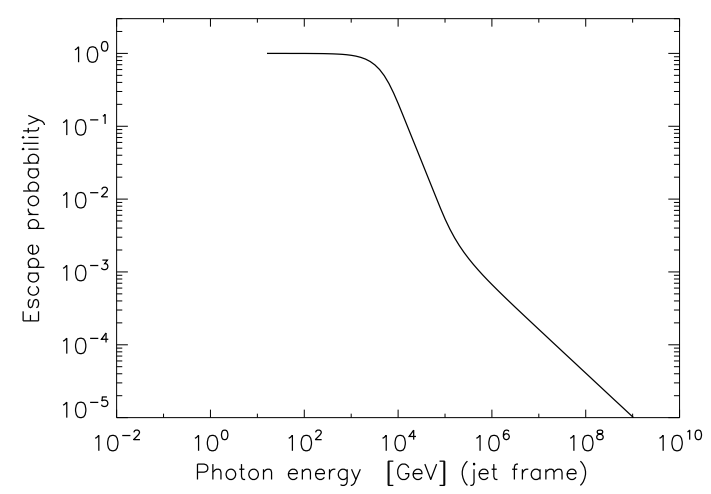

Figure 2: Escape probability for photons from the emission region of FSRQ PKS 1510-089 as a function of (jet frame) energy.

as luminosities instead of fluxes introduces a redshift bias due to the common redshift-dependence of the luminosities (e.g., [9]). This is particular prominent for samples that cover a wide distance range. On the other hand, intrinsic luminosity correlations can only be recovered in flux-flux diagrams when linear in its intrinsic quantities, and may be lost otherwise [3, 9]. Any artificial restriction of the dynamical flux/luminosity range in case of observational data by, e.g., finite instrument sensitivities, may also cause a serious overestimation of the strength of a correlation (e.g., [9]). Finally, selection biases may cause misleading correlation results. In the present work a correlation analysis is performed between neutrino number and photon number quantities of the model SEDs. In this sense biases that might be caused by instrument limitations and observational uncertainties do not play a major role. If any, only the selection procedure underlying the present sample may potentially impact.

A search for photon flux - neutrino flux correlations is performed using the Spearman rank order correlation coefficient $r_{S}$ and Kendall's $\tau$ (for the methology see, e.g., [9] \& references therein). Several photon and neutrino energy ranges are considered for this purpose (with view on applicability to presently available instruments): optical UBVRI-bands, $0.3-10 \mathrm{keV}, 15-150 \mathrm{keV}$, 0.1-100 GeV, 1-100 GeV and 0.1-10 TeV ranges in the photon domain, and $>0.01 \mathrm{PeV},>1 \mathrm{PeV}$ and $>10 \mathrm{PeV}$ in the neutrino domain. The inclusion of optical and X-ray bands in this analysis may test the importance of those parts of the target photon field on the neutrino emission.

Tab. 1 lists the results of this correlation analysis based on the 12 sample sources using photon and neutrino number fluxes. No convincing flux correlation is found between any optical and any neutrino bands. Essentially the same holds true for X-ray and neutrino bands, with the exception of a possible mild signal when considering $>10 \mathrm{PeV}$ neutrino fluxes which, however, vanishes when energy fluxes are used, indicating its likely spurious origin. Hence, the results of the flux-flux correlation analysis therefore do either not support a tight linear correlation between neutrino and photon luminosities in the considered energy bands, or indicate a high degree of scatter that hides any such correlation.

In case of luminosities a partial correlation analysis using the Spearman rank order correlation coefficient $r_{S}$ is used to account for the common redshift-dependence of the luminosities. In general 


\begin{tabular}{l|l||l|l||l|l}
\hline Photon band & $\begin{array}{l}\text { neutrino ener- } \\
\text { gies }\end{array}$ & $r_{s}$ & $\begin{array}{l}\text { chance } \\
\text { prob. }\end{array}$ & $\tau$ & $\begin{array}{l}\text { chance } \\
\text { prob. }\end{array}$ \\
\hline U-band & $>10 \mathrm{TeV}$ & 0.38 & 0.217 & 0.30 & 0.170 \\
B-band & $>10 \mathrm{TeV}$ & 0.31 & 0.319 & 0.24 & 0.273 \\
V-band & $>10 \mathrm{TeV}$ & 0.30 & 0.342 & 0.21 & 0.337 \\
R-band & $>10 \mathrm{TeV}$ & 0.24 & 0.443 & 0.18 & 0.411 \\
I-band & $>10 \mathrm{TeV}$ & 0.21 & 0.513 & 0.15 & 0.493 \\
U-band & $>10 \mathrm{PeV}$ & 0.43 & 0.159 & 0.33 & 0.131 \\
B-band & $>10 \mathrm{PeV}$ & 0.36 & 0.245 & 0.27 & 0.217 \\
V-band & $>10 \mathrm{PeV}$ & 0.35 & 0.265 & 0.24 & 0.273 \\
R-band & $>10 \mathrm{PeV}$ & 0.31 & 0.331 & 0.21 & 0.337 \\
I-band & $>10 \mathrm{PeV}$ & 0.26 & 0.417 & 0.18 & 0.411 \\
\hline $0.3-10 \mathrm{keV}$ & $>10 \mathrm{TeV}$ & 0.50 & 0.101 & 0.36 & 0.100 \\
$15-150 \mathrm{keV}$ & $>10 \mathrm{TeV}$ & 0.43 & 0.159 & 0.27 & 0.217 \\
$0.3-10 \mathrm{keV}$ & $>10 \mathrm{PeV}$ & 0.54 & 0.067 & 0.39 & 0.075 \\
$15-150 \mathrm{keV}$ & $>10 \mathrm{PeV}$ & 0.53 & 0.075 & 0.36 & 0.100 \\
\hline$>100 \mathrm{MeV}$ & $>10 \mathrm{TeV}$ & 0.22 & 0.484 & 0.15 & 0.493 \\
$>1 \mathrm{GeV}$ & $>10 \mathrm{TeV}$ & -0.14 & 0.665 & -0.03 & 0.891 \\
$>100 \mathrm{MeV}$ & $>10 \mathrm{PeV}$ & 0.43 & 0.167 & 0.30 & 0.170 \\
$>1 \mathrm{GeV}$ & $>10 \mathrm{PeV}$ & 0.021 & 0.948 & 0.06 & 0.784 \\
\hline$>100 \mathrm{GeV}$ & $>10 \mathrm{TeV}$ & -0.07 & 0.832 & 0.05 & 0.815 \\
$>100 \mathrm{GeV}$ & $>10 \mathrm{PeV}$ & 0.11 & 0.750 & 0.20 & 0.392 \\
& & & & &
\end{tabular}

Table 1: Correlation coefficients (Spearman rank $r_{s}$, Kendall's $\tau$ ) between photon and neutrino fluxes of the sample.

the partial correlation describes the relationship between two variables when the third variable is held constant. Since the present hadronic model does not use any external target photon fields for particle-photon interactions, the neutrino and photon number quantities are beamed by the same factor from the jet to the galaxy frame for all energy bands. This common beaming factor dependence of the luminosities in this case may cause a bias to the correlation analysis, which is also tested in this work.

In Tab. 2 the results of the partial correlation analysis between luminosities at various energy bands taking into account their common redshift-dependence are reported. Comparing these results with a corresponding analysis that ignores any such redshift-dependence shows that a strong common redshift-dependence is indeed present in the sample. On the other hand, applying the same procedure to test the strength of the common beaming factor dependence on any photon luminosityneutrino luminosity relation reveals no strong effects. This may be due to the very narrow range of Doppler factors used in the sample SEDs. No convincing correlation is found between any of the optical with any of the neutrino bands. The strength of the LE hump is in principle connected to neutrino production via the effective photomeson production probability. The non-detection of a significant correlation signal likely indicates a large degree of scatter in this relation for the present sample. A statistically weak signal is apparent when correlating the hard X-ray with any of the 


\begin{tabular}{l|l|l}
\hline Photon band & correlation coefficient & chance probability \\
\hline U-band & 0.468 & 0.151 \\
B-band & 0.486 & 0.134 \\
V-band & 0.464 & 0.155 \\
R-band & 0.468 & 0.151 \\
I-band & 0.334 & 0.325 \\
$0.3-10 \mathrm{keV}$ & 0.360 & 0.285 \\
$15-150 \mathrm{keV}$ & 0.674 & 0.021 \\
$>100 \mathrm{MeV}$ & 0.922 & $6 \cdot 10^{-6}$ \\
$>1 \mathrm{GeV}$ & 0.732 & $8 \cdot 10^{-3}$ \\
$>100 \mathrm{GeV}$ & 0.406 & 0.223 \\
\hline
\end{tabular}

Table 2: Spearman rank partial correlation coefficient between photon and neutrino ( $>1 \mathrm{PeV})$ luminosities of the sample.

neutrino bands. Note that in the present sample SEDs the hard X-ray band is associated either with the LE or HE hump. When correlating the soft X-ray band with any of the neutrino bands this weak signal vanishes. This may indicate that the impact of the synchrotron jet emission on the neutrino luminosity is again associated with large scatter within the present sample that prevents a significant correlation.

Correlating several bands of the HE hump with the neutrino luminosity reveals a convincing correlation for the $>100 \mathrm{MeV}$ photon regime (and in a somewhat reduced strength for $>1 \mathrm{GeV}$ ). This band encompasses the peak of the HE component in all the sample source SEDs, and is in all cases dominated by proton synchrotron radiation with an additional cascade contribution. Because the same protons that give rise to proton synchrotron radiation are also responsible for neutrino production in AGN jets, to some extent a connection between proton synchrotron dominated photon and neutrino emission is present. Noting in addition the well-known photon-neutrino relation between photon cascade and neutrino power results in an overall photon-neutrino correlation signal as noted above.

Moving up in photon energy, no tight correlation is found between the VHE ( $>100 \mathrm{GeV})$ and neutrino luminosity. The impact of photon absorption in the EBL at $\leq$ VHEs may contribute to explaining this finding. In all source SEDs in the present sample is the efolding cutoff energy for EBL-absorption at lower energies than the corresponding efolding energy for internal absorption/re-processing. Hence, some portion, depending on source redshift, of the photon luminosity is absorbed during propagation to Earth, while the neutrino luminosity remains unchanged in flavor sum. The situation improves only slightly when considering photon luminosities at source (i.e., EBL de-absorbed): a convincing correlation between the VHE photon and the neutrino bands could still not be found, likely due to large scatter. This makes the VHE band here unsuitable for utilizing the photon-neutrino connection of hadronic interactions.

Note that the results of the here presented correlation study are in general strongly dependent on the hadronic AGN emission model considered. 


\section{References}

[1] M.G. Aartsen et al (IceCube coll. Observation of High-Energy Astrophysical Neutrinos in Three Years of IceCube Data, Phys.Rev.Lett. 113 (2014) 101101Observation of High-Energy Astrophysical Neutrinos in Three Years of IceCube Data

[2] M. Böttcher, A. Reimer, K. Sweeney, A. Prakash, Leptonic and Hadronic Modeling of Fermi-detected Blazars, ApJ 768 (2013) 54

[3] E.D. Feigelson, C.J. Berg, X-ray observations of $203 C R$ radio galaxies and their environs, ApJ 269 (1983) 400

[4] J.D. Finke, S. Razzaque, C.D. Dermer, Modeling the Extragalactic Background Light from Stars and Dust, ApJ 712 (2010) 238

[5] F. Krauss, M. Kadler, K. Mannheim, et al., TANAMI blazars in the IceCube $>$ PeV-neutrino fields, $A \& A 566$ (2014) 7

[6] R. Moharana, S. Razzaque, Angular correlation of cosmic neutrinos with ultrahigh-energy cosmic rays and implications for their sources, JCAP, arXiv:1501.05158 (2015)

[7] A. Mücke, J.P. Rachen, R. Engel, R.J. Protheroe, T. Stanev, Photomeson production in astrophysical sources, Proc. 19th Texas Symposium on Relativistic Astrophysics and Cosmology, Paris, 1998; arXiv:9905153 (1999)

[8] A. Mücke, R. Engel, J.P. Rachen, R.J. Protheroe, T. Stanev, Monte Carlo simulations of photohadronic processes in astrophysics, CoPhC 124 (2000) 290

[9] A. Mücke, M. Pohl, P. Reich, W. Reich, et al., On the correlation between radio and gamma ray luminosities of active galactic nuclei, A\&A 321 (1997) 33

[10] A. Mücke, R.J. Protheroe, R. Engel, J.P. Rachen, T.Stanev, BL Lac objects in the synchrotron proton blazar model, APh 18 (2003) 593

[11] P. Padovani, E. Resconi, Are both BL Lacs and pulsar wind nebulae the astrophysical counterparts of IceCube neutrino events?, MNRAS 443 (2014) 474

[12] A. Reimer On the Physics of Hadronic Blazar Emission Models, J. Phys.: Conf. Ser. 355 (2012) 012011 